\title{
The radial velocities and physical parameters of HD 553^
}

\author{
R. Duemmler ${ }^{1}$, I. Kh. Iliev², and L. Iliev ${ }^{3}$ \\ 1 Astronomy Division, PO Box 3000, 90014 University of Oulu, Finland \\ 2 National Astronomical Observatory (NAO) Rozhen, and Isaac Newton Institute of Chile, Bulgarian Branch, PO Box 136, \\ 4700 Smolyan, Bulgaria \\ 3 Institute of Astronomy, Bulgarian Academy of Sciences, and Isaac Newton Institute of Chile, Bulgarian Branch, Tsarigradsko \\ Shosse Blvd. 72, 1184 Sofia, Bulgaria
}

Received 3 June 2002 / Accepted 5 September 2002

\begin{abstract}
HD 553 was discovered as an eclipsing binary by Hipparcos. Here, we present the first radial velocity curve for this system. It is found, that HD 553 is a double-lined spectroscopic binary. Despite the large luminosity difference, the two components of this system are of very similar mass. The primary, a K0-giant, fills a large fraction of its Roche-lobe. The secondary is, despite its very similar mass, still a late-type dwarf. The radial velocity curve allows to constrain several stellar and system parameters.
\end{abstract}

Key words. stars: individual: HD 553 - binaries: spectroscopic - binaries: eclipsing - stars: late-type - stars: starspots techniques: radial velocities

\section{Introduction}

Additional to the astrometric parameters of positions, proper motions and trigonometric parallaxes, the highly successful Hipparcos mission also provided photometric information for the target stars. Thus, a large number of new variables has been discovered by Hipparcos. Among these is HD 553 (=HIP 834; $\alpha_{2000}=00^{\mathrm{h}} 10^{\mathrm{m}} 10^{\mathrm{s}} 4647, \delta_{2000}=+64^{\circ} 38^{\prime} 48^{\prime \prime} \cdot 174 ; V \approx 88^{\mathrm{m}} 2$ ). Kazarovets et al. (1999) subsequently classified the system as an eclipsing binary of the $\beta$ Lyr type (i.e. two minima are present and there is no constant part due to the ellipticity effect) and gave it the name V 741 Cas. Strassmeier et al. (2000) identified HD 553 as a single-lined spectroscopic binary and included it into their list of potential targets for Doppler-imaging from their survey of stellar activity indicators.

The Hipparcos catalogue (ESA 1997) provides the following information about this system:

- $V=8$ 8. $^{\mathrm{m}} 11, B-V=1^{\mathrm{m}} \cdot 032 \pm 0.016, V-I=1^{\mathrm{m}} \cdot 00 \pm 0.01$

- $\pi=4.74 \pm 0.83$ mas

- period $P=9.0576 \pm 0.0008$ days, $T_{0}=2448504.0610$ as time of zero phase (from fits to the Tycho-lightcurve).

HD 553 is an ideal target for Doppler imaging. At observatories north of latitude $26^{\circ}$, like the NAO Rozhen, Bulgaria $\left(\varphi \approx 42^{\circ}\right)$, it is circumpolar, allowing for in principle continuous observations. This would allow to follow the spot evolution on any timescale (longer than the rotation period) without the often

Send offprint requests to: L. Iliev,

e-mail: liliev@libra.astro.bas.bg

* Based on observations made with the Coudé-spectrograph at the $2 \mathrm{~m}$-RCC-telescope of the National Astronomical Observatory Rozhen, Bulgaria.
1 yr timegap between subsequent maps for other Doppler imaging targets. Assuming that the orbital and rotational axes of the eclipsing binary are aligned, the inclination of the rotational axis is high allowing to observe most of the stellar surface. It is bright enough to allow for good signal-to-noise spectra within a reasonably short time. Because the eclipse, when the primary is in the back, is far from total we can also resolve most of the stellar surface during the eclipse. Its projected rotational velocity $v \sin i=42.5 \mathrm{~km} \mathrm{~s}^{-1}$ (see Sect. 3) is high enough to allow for reliable Doppler imaging even with a moderate resolution of about 30000 , as obtainable with the Coudé spectrograph at Rozhen. Finally, the period of 9 days is long enough that one good spectrum per observing night is enough to provide a good phase coverage, and thus the object can easily be included in any spectroscopic night's object list. On the other hand, the period is short enough to allow the data for a complete map to be collected within the typical $1-2$ week spectroscopic observing runs.

Therefore, we became interested in this object and started an observing campaign. The first step for reliable Doppler imaging is to find a good radial velocity curve. The problem of Doppler imaging is ill-posed and has a lot of free parameters. Thus, any additional constraints that can be put prior to Doppler imaging is of great value. Since Doppler imaging relies on distortions in the line profiles caused by the surface structures, it is important to remove any other distortion of the line profiles, in particular any shift of the lines with respect to the same lines at other phases. In principle, the radial velocity shifts from the orbital motions could be removed prior to Doppler imaging by simply cross-correlating the spectra. However, the spots on the stellar surface introduce systematic errors in the shifts obtained in this way. It is therefore useful to determine the radial velocity 
based on a large time scale; each individual radial velocity will still be influenced by the line profile distortions, but the limited lifetime of the spots ensures that spectra at the same phases taken a long time apart average out these distortions. Also, any reliable determination of the orbital parameters is based on a good value for the orbital period (all other parameters more or less correlate with the orbital period), which becomes more and more accurate the longer the time base of the radial velocity curve.

Here, we report on the first radial velocity curve of the HD 553 system based on 20 high-accuracy radial velocities obtained over the time base of 2 years, and the conclusions about the system and stellar parameters that can be drawn from it.

\section{Observations and reductions}

The observing material consists of 20 spectra obtained with the Coudé spectrograph at the 2m-RCC-telescope of the National Astronomical Observatory Rozhen, Bulgaria. The data were collected in 4 observing runs in October/November 1999, April and October 2000, and September/October 2001.

The grating 632/22:3 and an entrance slit width of $300 \mu \mathrm{m}$ were used, resulting in a resolution of about 30000 . The spectra were recorded with the Photometrics AT200 camera on a $1024 \times 1024 \mathrm{CCD}$ with pixel size $24 \times 24 \mu \mathrm{m}^{2}$, yielding a spectral range of about $10 \mathrm{~nm}$ for each spectrum. In different seasons, different spectral regions have been observed: in 1999 , the region around the $\mathrm{Ca}$ I line at $643.9 \mathrm{~nm}$, in 2000 and 2001, the region around the $\mathrm{Fe}$ I line at $617.3 \mathrm{~nm}$ have been observed; additionally, in 2001, another region, that around a group of telluric lines at $630 \mathrm{~nm}$, was observed immediately before or after the Fe I-region, which allows an independent check of the wavelength scale via the atmospheric lines and of the radial velocities $(R V \mathrm{~s})$ via the comparison of the $R V \mathrm{~s}$ from the different regions at virtually the same time. The average signal-to-noise ratio of the spectra is 136 .

Additional to the spectra of HD 553, several spectra of the $R V$ standard $\beta$ Gem have been obtained in each observing run, except for Oct. 2000, when we observed the $R V$ standard $\alpha$ Cas. Both stars are classified as K0 III, and are therefore suitable templates for HD 553.

The information for each spectrum is given in Table $1^{1}$.

Each observed stellar spectrum is accompanied by a pair of comparison spectra (one taken before, the other after the stellar exposure) and a number of flatfield images. In several nights, a large number of bias-images was obtained, which were found to be unchanging within an observing run; they were averaged, then subtracted from each image of the same run.

The reduction of the material has been done using the spectrum reduction package $4 \mathrm{~A}$ (Ilyin 2000), and consists of the steps bias image subtraction, flatfielding, scattered light subtraction, optimal spectrum extraction, wavelength calibration.

The wavelength calibration is based on the comparison spectrum images consisting of Th-Ar emission lines. Each

\footnotetext{
1 Table 1 is also available in electronic form at the CDS via anonymous ftp to cdsarc.u-strasbg.fr (130.79.128.5) or via http://cdsweb.u-strasbg.fr/cgi-bin/qcat?J/A+A/395/885
}

Th-Ar-image consists of a pair of comparison spectra: one is above, the other below the area covered by the stellar spectrum, but overlapping with it. This allows to take into account a slope of the lines of constant wavelength across the stellar spectrum. The observation of a comparison image before and another one after the stellar exposure allows to take into account a possible systematic drift of the pixel coordinate system along the wavelength axis. Thus, the final dispersion curve of each stellar image is based on a total of $4 \mathrm{Th}$-Ar-comparison spectra, and is interpolated to the central position of the stellar spectrum and the time of mid-exposure of the stellar spectrum. The dispersion curve is approximated by a polynomial of degree 3 (except for Sept./Oct. 2001, where a degree of 5 was necessary) and has typically an accuracy of $0.02-0.04 \mathrm{~km} \mathrm{~s}^{-1}$.

After the wavelength calibration, the spectra are transformed into heliocentric wavelengths and normalized to the apparent continuum which is a polynomial of degree 4 approximating the high points of the stellar spectrum.

It is worth mentioning that $4 \mathrm{~A}$ estimates the errors of each pixel in the original image (after bias subtraction) assuming Poissonian noise and propagates these errors through each step of the reduction procedure. In this way, reliable errors are available for each pixel in the final spectrum in both flux and wavelength resulting also in individual errors for each RV.

\section{Radial velocity measurements}

Radial velocities were determined for each spectrum by crosscorrelation with the average of all spectra of the radial velocity standard observed during the same observing run. For this, the spectra of the standards were artificially spun up to different values of the projected rotational velocity $v \sin i$, and the spectrum which maximizes the cross-correlation peak height gives the best value of $v \sin i$. This was done for both standards $\beta \mathrm{Gem}$ and $\alpha$ Cas and for all spectra of HD 553. The result is $v \sin i=42.5 \pm 0.5 \mathrm{~km} \mathrm{~s}^{-1}$, independent of which standard is used, independent of the spectral region and of the phase in the binary orbit. The latter allows the conclusion that the ellipticity of the primary in the binary (the giant) is not very pronounced.

Before cross-correlating the artificially broadened spectra of the templates with the spectra of HD 553 they were corrected for the radial velocity of the corresponding standard star. For $\beta \mathrm{Gem}, R V=+(3.3 \pm 0.1) \mathrm{km} \mathrm{s}^{-1}$ according to the Astronomical Almanach for 2002 (2001) and Stefanik et al. (1999). For $\alpha$ Cas, in recent publications several different $R V$ s are given, casting some doubt on its suitability as a radial velocity standard: $R V=-(3.9 \pm 0.1) \mathrm{km} \mathrm{s}^{-1}$ according to the Astronomical Almanach for 2002 (2001), $R V=-(4.3 \pm 0.2) \mathrm{km} \mathrm{s}^{-1}$ according to Udry et al. (1999), and $R V=-(4.79 \pm 0.04) \mathrm{km} \mathrm{s}^{-1}$ according to de Medeiros \& Mayor (1999). Our investigation (see Sect. 4) shows that the value by de Medeiros \& Mayor (1999) is best. Note, that by observing the standard stars in the same run as the spectra of HD 553 (usually immediately before or after HD 553) and reducing them in exactly the same way, then moving them to $R V=0$ using the values from the literature, the $R V \mathrm{~s}$ of HD 553 are on the system of the literature velocities of the standards, without any contribution from some systematic 


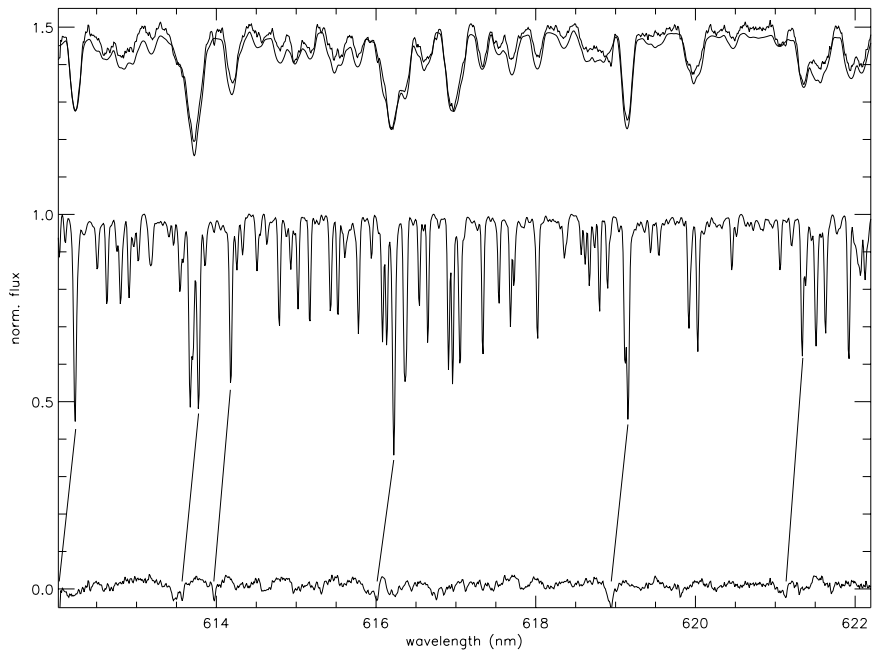

Fig. 1. The spectrum of HD 553 (thick line, top) compared to the spun-up spectrum of $\beta$ Gem ( $v \sin i=42.5 \mathrm{~km} \mathrm{~s}^{-1}$; thin line), both shifted up by 0.5. At the bottom is the difference between these two spectra, showing some weak, narrow absorption lines of the secondary. The central spectrum is the original spectrum of $\beta \mathrm{Gem}$, used to measure the $R V$ of the secondary, which is here $-101 \mathrm{~km} \mathrm{~s}^{-1}$ with respect to the primary (HJD 2452185.5178). The spectra of HD 553 are in the restframe of the primary (determined from a preliminary orbit fit, based only on the primary's $R \mathrm{Vs}$ ).

velocity zero-points (which could be different in different runs) (see also Sect. 4).

We also searched for signs of the secondary in the spectra. Indeed, a faint spectrum displaying narrow lines moving in antiphase to the strong, broad lines of the giant were found, after the spectrum of $\beta$ Gem had been subtracted from that of HD 553. The $R V \mathrm{~s}$ from these lines were measured by crosscorrelation with the original (not spun-up) spectrum of the $R V$ standard of that run. $R V \mathrm{~s}$ of the secondary are not measureable when the secondary is eclipsed. Figure 1 shows the spectrum of HD 553 in the $617.3 \mathrm{~nm}$ region and identifies some lines of the secondary. It can be seen that the continuum of the broadened $\beta$ Gem spectrum tends to be below that of HD 553. The reason is the imperfect continuum normalization for HD 553, which is due to the very low signal-to-noise and to the fact that its (pseudo-)continuum is only defined by a very few regions without broad, blended features. However, the difference spectrum is mostly flat so that $R V$ measurements should not be influenced and no attempt has been made to correct for this. Note that some "emission" features appear in the difference spectrum which result from an imperfect match of some of the features in the artificially broadened $\beta$ Gem spectrum and in the HD 553 spectrum. Yet, the lines of the secondary are clearly visible and measureable.

All the strong lines in the $\beta$ Gem spectrum are represented in the spectrum of the secondary; several weaker features are also identifiable. The lines are narrow, which can be seen e.g. with the clearly resolved double feature near $613.5 \mathrm{~nm}$. This indicates a much lower $v \sin i$ of the secondary; together with the low luminosity and the presence of the same strong lines as in the spectrum of the late giant $\beta \mathrm{Gem}$, this makes the secondary a late-type dwarf. Therefore, we decided to use the unbroadened spectum of $\beta$ Gem as a template for the $R V$ measurements of the secondary. Earlier experience has shown that a good $R V$ of $\beta$ Gem can be measured with the Sun as a template. So, a mismatch of the spectral types of $\beta$ Gem and of the secondary should have only a small effect on the final $R V$ of the secondary.

Unfortunately, the lack of proper standards, the comparitively low signal-to-noise ratio in the spectrum of the secondary, and the also visible systematics resulting from the not perfect match of the broadened $\beta$ Gem spectrum and the HD 553 spectrum make it impossible to analyse the spectrum of the secondary in more detail.

All $R V \mathrm{~s}$ are given in Table 1 together with their errors as determined by 4A. These take into account the errors of the pixels in the spectra in the overlapped region of both the programme and the standard spectrum as well as the height and curvature of the cross-correlation maximum (for details, see Ilyin 2000).

\section{Orbital solution}

The radial velocities in Table 1 were used in a double-lined orbit fit. A few details about the fit routine can be found in Duemmler et al. (1997). The weights of the individual $R V \mathrm{~s}$ were obtained as the inverse variances, based on the $R V$ errors. However, it turned out, that the primary and secondary had very different values of $\chi^{2}$; therefore, the weights of the secondary were reduced by a common factor $\chi_{1}^{2} / \chi_{2}^{2}=0.108$.

While the large ratio of the $\chi^{2}$ values of the primary and secondary have shown that the errors for the secondary given in Table 1 are clearly underestimated, the overall reduced $\chi^{2}$ of the fit of 2.1 is quite good. Although the formal probability of exceeding a value of 2.0 is just $1 \%$ for 15 degrees of freedom, experience with many fits has shown that, even for larger numbers of $R V \mathrm{~s}$ with smaller errors, values of 2-3 for $\chi^{2}$ are not uncommon. However, larger deviation than expected from the errors may also result from stellar activity: starspots distort the line profiles, and cause the $R V$ to deviate systematically. Therefore, we have investigated the $\mathrm{O}-\mathrm{C}$ values as a function of phase and observing run, but we do not find any systematic (see Fig. 2). One should, however, keep in mind that 20 measurements is still a rather small statistics.

The errors of the fit-parameters were determined by 10000 bootstrap runs (see Duemmler et al. 1997 and, e.g., Efron \& Tibshirani 1993). To each $R V$ computed from the orbital solution we add a randomly chosen value taken from the $\mathrm{O}-$ $\mathrm{C}$ values. Since on average low weighted $R V \mathrm{~s}$ tend to have larger $\mathrm{O}-\mathrm{C}$, each $\mathrm{O}-\mathrm{C}$ is accompanied by its original weight; the choice of $\mathrm{O}-\mathrm{C}$ values is done with replacement, i.e. the same O-C can be chosen several times while others are not used. With this artificial data set a new orbital fit is performed, leading to a slightly different set of parameters than the fit to the original data. The error of a parameter is given by the standard deviation of this parameter from all the bootstrap samples. The resulting orbital and derived parameters are presented in Table 2. The orbit is plotted in Fig. 2.

Strassmeier et al. (2000) have also measured two $R V$ s for HD 553. While these two $R V \mathrm{~s}$ increase the time span of the $R V$ curve by another year, the large errors compared to our $R V \mathrm{~s}$ 
Table 1. The radial velocities of HD 553. The columns are: the heliocentric Julian date (HJD-2450000) of the mid-exposure, the approximate centre of the spectral region in $\mathrm{nm}$, the signal-to-noise ratio $(S N R)$ of the spectrum, the radial velocities and errors of the primary and secondary, respectively, in $\mathrm{km} \mathrm{s}^{-1}$.

\begin{tabular}{crrrl}
\hline \hline HJD-2450000 & $\lambda_{\mathrm{c}}$ & $S N R$ & $R V_{1}$ & $R V_{2}$ \\
\hline 1478.6010 & 644 & 76 & $17.26 \pm 0.38$ & $-97.45 \pm 0.77$ \\
1481.3821 & 644 & 162 & $-88.47 \pm 0.38$ & $11.87 \pm 0.66$ \\
1482.4879 & 644 & 157 & $-102.36 \pm 0.26$ & $25.36 \pm 0.69$ \\
1483.3475 & 644 & 189 & $-89.04 \pm 0.23$ & $12.81 \pm 0.55$ \\
1484.3707 & 644 & 151 & $-51.71 \pm 0.20$ & - \\
1485.3671 & 644 & 174 & $-9.59 \pm 0.23$ & $-70.83 \pm 0.54$ \\
1648.5779 & 617 & 73 & $-3.36 \pm 0.40$ & $-74.37 \pm 0.69$ \\
1652.3007 & 617 & 59 & $-40.38 \pm 0.32$ & - \\
1831.3489 & 617 & 160 & $23.94 \pm 0.35$ & $-103.73 \pm 0.53$ \\
1832.2459 & 617 & 121 & $8.41 \pm 0.36$ & $-89.52 \pm 0.68$ \\
1832.6138 & 617 & 182 & $-3.91 \pm 0.34$ & $-77.18 \pm 0.50$ \\
1833.3567 & 617 & 160 & $-33.31 \pm 0.29$ & - \\
1834.3547 & 617 & 169 & $-74.61 \pm 0.36$ & $-0.42 \pm 0.53$ \\
2181.4355 & 630 & 120 & $-73.98 \pm 0.44$ & $-3.81 \pm 1.63$ \\
2181.4664 & 617 & 125 & $-71.79 \pm 0.26$ & $-3.30 \pm 0.54$ \\
2182.5348 & 630 & 126 & $-28.18 \pm 0.33$ & - \\
2182.5675 & 617 & 147 & $-26.87 \pm 0.19$ & - \\
2183.5393 & 630 & 102 & $10.27 \pm 0.45$ & $-89.74 \pm 1.18$ \\
2185.4905 & 630 & 145 & $11.20 \pm 0.36$ & $-90.93 \pm 1.06$ \\
2185.5178 & 617 & 132 & $10.69 \pm 0.27$ & $-90.48 \pm 0.37$ \\
\hline & & & &
\end{tabular}

lead to very small weights in the fit. The result is identical to the fit presented in Table 2, except that the errors of the parameters even increased slightly. We therefore decided to ignore the Strassmeier et al. measurements.

We also attempted to fit an eccentric orbit. The result $e=$ $0.0016 \pm 0.0023$ with $\omega=350^{\circ} .7 \pm 0^{\circ} .1$ clearly shows that the orbit is circular. The Lucy-Sweeney $F$-test (Lucy \& Sweeney 1971; Lucy 1989) gives a $96.2 \%$ probability for a circular orbit.

Another fit allowed for different $\gamma$ velocities for the 4 observing runs, while forcing all other parameters to be the same for the 4 data sets. That means that we are looking for systematic $R V$ errors between the different runs, or for systematic changes of the $\gamma$ velocity from run to run, which could be caused by a third body. Within the errors, the orbital parameters do not change compared to those given in Table 2; the results for $\gamma$ are:

$$
\begin{aligned}
\text { Oct./Nov. } 1999 & \gamma_{1}=-39.27 \pm 0.19 \\
\text { Apr. } 2000 & \gamma_{2}=-38.62 \pm 0.34 \\
\text { Oct. } 2000 & \gamma_{3}=-38.73 \pm 0.25 \\
\text { Sep./Oct. } 2001 & \gamma_{4}=-38.75 \pm 0.26
\end{aligned}
$$

The largest difference, $\gamma_{1}-\gamma_{2}$, is only $1.7 \sigma$, i.e. there is no indication for any significant change of $\gamma$ over the two-year interval or for a significant error in the velocity zero points of the different runs. It is noteworthy that $\gamma_{3}$ is based on $R V \mathrm{~s}$ measured with $\alpha$ Cas as standard, for whose $R V$ we adopted the value given by de Medeiros \& Mayor (1999). Would we have
Table 2. The orbital parameters of the double-lined spectroscopic binary HD 553. Errors of the parameters are based on 10000 bootstrap runs. The primary derived parameter is $P_{\text {rest }}$, the period in the restframe of the binary system, i.e. the observed period corrected for the Doppler-effect caused by $\gamma$. All dynamical derived parameters are based on $P_{\text {rest }}$, while the epochs $T_{0}, T_{\text {conj }}$ are in the observer's system, i.e. based on $P_{\mathrm{obs}} . T_{0}$ is the time of maximum $R V$ for the primary (the giant), $T_{\text {conj }}$ is the conjugation time with the primary in the back. $\chi^{2}$ is the reduced $\chi^{2}$ of the fit, i.e. the weighted sum of squared deviations, divided by the number of degrees of freedom. $\sigma$ is the mean standard deviation of a single $R V$ with average weight, $\sigma_{1}$ and $\sigma_{2}$ are the same for the primary and secondary, respectively.

\begin{tabular}{lc}
\hline \hline parameter & \\
\hline$K_{1}\left(\mathrm{~km} \mathrm{~s}^{-1}\right)$ & $63.32 \pm 0.19$ \\
$K_{2}\left(\mathrm{~km} \mathrm{~s}^{-1}\right)$ & $66.04 \pm 0.41$ \\
$P_{\text {obs }}($ days $)$ & $9.05997 \pm 0.00011$ \\
$\gamma\left(\mathrm{km} \mathrm{s}^{-1}\right)$ & $-38.93 \pm 0.12$ \\
$T_{0}(\mathrm{HJD})$ & $2451813.0877 \pm 0.0036$ \\
& \\
$P_{\text {rest }}($ days $)$ & $9.06115 \pm 0.00011$ \\
$q=m_{2} / m_{1}$ & $0.9589 \pm 0.0066$ \\
$a_{1} \sin i\left(R_{\odot}\right)$ & $11.336 \pm 0.034$ \\
$a_{2} \sin i\left(R_{\odot}\right)$ & $11.822 \pm 0.073$ \\
$m_{1} \operatorname{sin~} i\left(M_{\odot}\right)$ & $1.037 \pm 0.013$ \\
$m_{2} \operatorname{sin~} i\left(M_{\odot}\right)$ & $0.995 \pm 0.009$ \\
$T_{\text {conj }}(\mathrm{HJD})$ & $2451815.3527 \pm 0.0035$ \\
$\chi^{2}$ & 2.14 \\
$\sigma\left(\mathrm{km} \mathrm{s}^{-1}\right)$ & 0.56 \\
$\sigma_{1}\left(\mathrm{~km} \mathrm{~s}^{-1}\right)$ & 0.59 \\
$\sigma_{2}\left(\mathrm{~km} \mathrm{~s}^{-1}\right)$ & 0.97 \\
\hline
\end{tabular}

adopted the other values given in the literature, $\gamma_{3}$ would deviate by $0.9 \mathrm{~km} \mathrm{~s}^{-1}$ (Astronomical Almanach for 2002 (2001)) and $0.5 \mathrm{~km} \mathrm{~s}^{-1}$ (Udry et al. 1999), respectively; it is clear, that the value by de Medeiros \& Mayor (1999) is best, at least at the time of our measurements, Oct. 2000.

It is remarkable that the orbital parameters in Table 2 show a system with almost identical masses for the two stars, although one of the stars is clearly evolved.

It should be mentioned that our period $P_{\mathrm{obs}}$ is longer than both the period given in the Hipparcos catalogue (ESA 1997; $P=9.0576 \pm 0.0008)$ and the period given by Strassmeier et al. (2000; $P=9$ d.0580). While Strassmeier et al.'s period is consistent with the one given by Hipparcos, our period is longer by $3 \sigma$. However, since the Hipparcos period is based on a curve fit, whose shape is not specified, we feel that our period is more reliable.

\section{Conclusions and discussion}

We have found the Hipparcos-detected eclipsing binary HD 553 to be a double-lined spectroscopic binary with a giant as primary and a faint companion of almost equal mass. From 20 high-quality $R V$ s collected over the course of 2 years, 

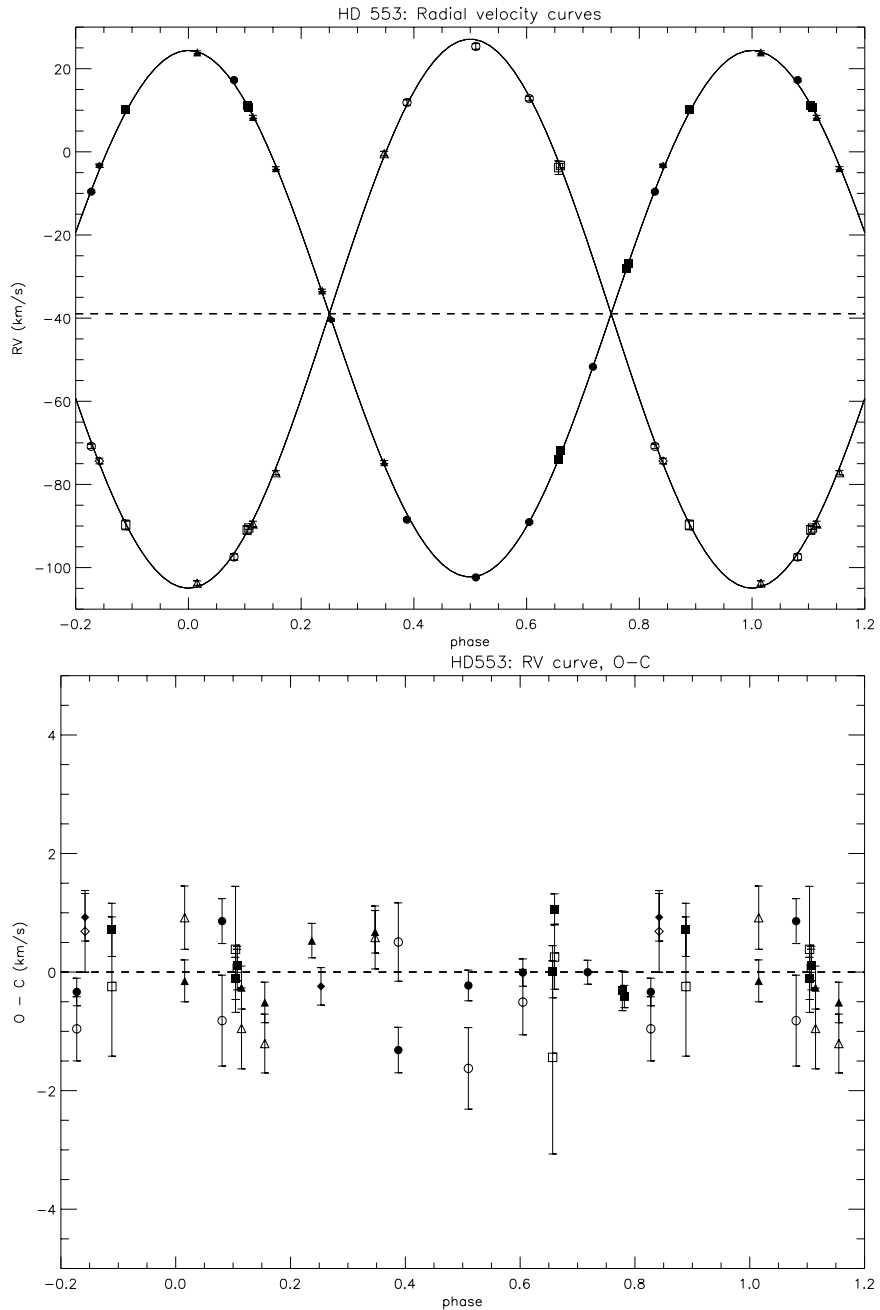

Fig. 2. Top: The radial velocity curves of the two stars in the HD 553 system. Filled symbols are used for the primary (the giant), open symbols for the secondary; circles denote the data for Oct./Nov. 99, diamonds those for Apr. 00, triangles those for Oct. 00, and squares those for Sep./Oct. 01. Errorbars are typically smaller than the symbol size. Bottom: O-C vs. phase.

the radial velocity curve was derived, leading to an improvement of the binary period and the derivation of other orbital and physical parameters.

If we assume that the primary is in synchronised rotation (i.e. the system is old and the primary has been a giant for a long time), the rotation and orbital axes should also be aligned, i.e. $P_{\text {rot }}=P_{\text {orb }}$, for which the period $P_{\text {rest }}$ in the rest of frame of the system should be used and $i_{\text {rot }}=i_{\text {orb }}=i$. Then, the projected radius is given by $v \sin i$ via

$R_{1} \sin i=\frac{P v \sin i}{2 \pi}=(7.608 \pm 0.090) R_{\odot}$,

with $v \sin i=(42.5 \pm 0.5) \mathrm{km} \mathrm{s}^{-1}$ as determined in Sect. 3 .

The assumption of aligned axes and synchronisation of rotation and orbit could, however, be wrong. While it is true that the system is old for the primary to become a giant by now, so possibly the axes are aligned, the periods may be different. During the expansion of the primary towards the giant branch the rotation period should increase due to angular momentum conservation. A longer rotation period would increase the radius given above. It would also imply that the primary's expansion happened quite recently, so that the tidal forces were not able to speed up again the primary's rotation. This is highly likely, because the secondary is still a dwarf, despite it having nearly the same mass as the primary.

Therefore, it is good to compare this lower limit for the radius with the radius that can be obtained from the photometric properties. According to Strassmeier et al. (2000), $V=8 \mathrm{~m} 11$, $B-V=1$ m $03, T_{\text {eff }}=4790 \mathrm{~K}$; and according to the Hipparcos catalogue (ESA 1997), $\pi=4.74$ mas, corresponding to a distance of $211 \mathrm{pc}$. The $V$-magnitude could be too faint, if the star has been covered by spots at the time of the measurements and $B-V$ could be too red; additionally, the interstellar extinction $\left(A_{V}, E_{B-V}\right)$ is unknown. This means, that the resulting absolute magnitude $M_{V}=1.59$ is an upper limit. With $B C=0.43$ (read off from Fig. 10.16, p. 197, in Gray 1992), the upper limit for the bolometric absolute magnitude becomes $M_{\text {bol }}=1 \mathrm{~m} .03$, and the lower limit for the luminosity $29 L_{\odot}$, leading to a lower limit of $7.85 R_{\odot}$ for the radius of the primary. This value is consistent with the lower limit from Eq. (2). Based on the good subtraction of the $\beta$ Gem spectrum from that of HD 553, we could repeat the calculation using the temparature of $\beta \mathrm{Gem}$ : $T_{\text {eff }}=4865 \mathrm{~K}$ (Drake \& Smith 1991$)$; this leads to $\left(7.61 R_{\odot}\right)$, identical to the lower limit in Eq. (2).

While both results are lower limits, it would be quite a coincidence, if the difference of the rotation and orbital periods and the projection factor $\sin i$ on the one hand and the spot coverage and interstellar absorption on the other lead to the same change in the radius. It is more likely, that $\sin i_{\text {rot }} \approx \sin i_{\text {orb }} \approx 1$, $P_{\text {rot }} \approx P_{\text {orb }}, V \approx V_{\text {unspotted }}$, and $A_{V} \approx 0$ (despite a galactic latitude of just $2^{\circ}$ ).

The effective radius of the Roche-lobe of the primary, $R_{\mathrm{RL}}$, i.e. the radius of the sphere having the same volume as the Roche-lobe, is another quantity with which to compare the lower limit for the primary's radius. According to Eggleton (1983)

$$
\begin{aligned}
R_{\mathrm{RL}} \sin i & =a \sin i \frac{0.49 Q^{2 / 3}}{0.6 Q^{2 / 3}+\ln \left(1+Q^{1 / 3}\right)} \\
& =(8.859 \pm 0.034) R_{\odot}
\end{aligned}
$$

with the inverse mass ratio $Q=m_{1} / m_{2}=1.0429 \pm 0.0072$.

The fact that the Roche-lobe radius is only about $1 R_{\odot}$ larger than the radius of the primary shows that the assumption of synchronisation cannot be grossly wrong: if the rotation period were longer than 10.6 (in the restframe of the primary), the star would overfill its Roche-lobe and most likely form a shell around the whole system, which seems not to be the case. Thus, the deviation from synchronicity cannot be larger than $16 \%$.

If we consider synchronous rotation as valid, the star fills a considerable fraction $(63.3 \%$ of the volume) of its Rochelobe. Still, since the star is nearly $40 \%$ smaller than the Roche lobe, we would not expect at present a large deviation from sphericity (see also Sect. 3, where it was found that $v \sin i$ does not depend on phase). According to Gray (1992, Appendix B), a $\mathrm{K} 0$ III star has on average a radius of $R(\mathrm{~K} 0 \mathrm{III})=11 R_{\odot}$. 
Either the primary in HD 553 is still expanding, in which case it will soon (in evolutionary terms) fill its Roche-lobe and start losing mass towards the secondary, or its inclination makes $R \sin i=7.6 R_{\odot}$ so much smaller than the typical radius, in which case the inclination would be about $44^{\circ}$. Since the system is eclipsing, the latter possibility seems highly unlikely; given the similar masses of the two components, but the very different luminosities, the former possibility seems to be very plausible. Thus, we expect HD 553 to become soon a semidetached system.

Finally, the small errors in the radial velocity curve presented here, based on the long time span of 2 years, should allow a good transformation of future spectra into the restframe of the primary for surface imaging. However, many RS CVn stars show persistent active longitudes with activity cycles lasting several years (Berdyugina \& Tuominen 1998). Thus, the relatively small amount of statistics, based on only 20 measurements over the comparatively short time span of 2 years may not be enough to ensure that all systematic effects from spots on the radial velocity curve are removed yet. Future spectra will therefore also be used to further improve the orbital parameters.

Acknowledgements. We thank the referee, Dr. Holger Lehmann, for his careful reading of the original version of the paper and for his useful questions and suggestions, which helped to improve the paper. A large part of this work was financially supported by the Academy of Finland and the Bulgarian Academy of Sciences through travel grants for RD and LI, for which we are grateful. We made use of the SIMBAD database, maintained at the Centre de Données Stellaire (CDS) in Strasbourgh, France.

\section{References}

The Astronomical Almanach for the year 2002, U.S. Government Printing Office, Washington, and The Stationary Office, London, 2001, p. H 42

Berdyugina, S. V., \& Tuominen, I. 1998, A\&A, 336, L25

de Medeiros, J. R., \& Mayor, M. 1999, A\&AS, 139, 433

Drake, J. J., \& Smith, G. 1991, MNRAS, 250, 89

Duemmler, R., Ilyin, I. V., \& Tuominen, I. 1997, A\&AS, 123, 209

Efron, B., \& Tibshirani, R. J. 1993, An Introduction to the Bootstrap (Chapman \& Hall, New York)

ESA 1997, The Hipparcos Catalogue, ESA SP-1200 (available at CDS, http://cdsweb.u-strasbg.fr/cgi-bin/ VizieR-2?-source=I/239)

Eggleton, P. P. 1983, ApJ, 368, 368

Gray, D. F. 1992, The observation and analysis of stellar photospheres (Cambridge Univ. Press, 2nd ed., Cambridge)

Ilyin, I. V. 2000, Ph.D. Thesis, University of Oulu, Finland

Kazarovets, E. V., Samus, N. N., Durlevich, O. V., et al. 1999, IBVS, 4659

Lucy, L. B. 1989, Obs., 109, 100

Lucy, L. B., \& Sweeney, M. A. 1971, AJ, 76, 544

Stefanik, R. P., Latham, D. W., \& Torres, G. 1999, in Precise Stellar Radial Velocities, IAU Coll. 170, ed. J. B. Hearnshaw, \& C. D. Scarfe), Astronomical Society of the Pacific, San Francisco, ASP Conf. Ser., 185, 354

Strassmeier, K. G., Washuettl, A., Granzer, T., et al. 2000, A\&AS, 142,275

Udry, S., Mayor, M., Maurice, E., et al. 1999, in Precise Stellar Radial Velocities, IAU Coll. 170, ed. J. B. Hearnshaw \& C. D., Scarfe, Astronomical Society of the Pacific, San Francisco, ASP Conf. Ser., 185,383 